\title{
The forward peak for neutral projectiles
}

\author{
D H Jakubaßa-Amundsen \\ Physics Section, University of Munich, 8046 Garching, West Germany
}

Received 5 June 1989

\begin{abstract}
The capture of target electrons into the continuum of fast projectiles is calculated with the peaked impulse approximation. The electronic final state is taken as the low-energy scattering solution of the Schrödinger equation containing a short-range model potential. It is found that a forward peak indeed exists for neutral projectiles; it is, however, considerably broader than for charged projectiles. As an example, hydrogen and helium projectiles are considered, colliding with a neon target.
\end{abstract}

\section{Introduction}

Electrons captured by charged projectiles exhibit in their spectrum a cusp-like structure when the electron momentum $\boldsymbol{k}_{\mathrm{f}}$ matches the projectile velocity $\boldsymbol{v}$. This forward divergence of the theoretical differential cross section arises from the long-range nature of the Coulomb field acting between the electron and the projectile, which causes a $k^{-1 / 2}$ behaviour of the normalisation constant of the electronic Coulomb wave for $k \rightarrow 0$, where $k=\left|\boldsymbol{k}_{\mathrm{f}}-\boldsymbol{v}\right|$ is the electron momentum in the projectile reference frame (Macek 1970). Short-range potentials, on the other hand, which decrease asymptotically faster than $r^{-2}$ with distance, and which do not support zero-energy resonance states, should lead to a finite normalisation constant of the electronic continuum eigenfunction. Thus, in general, no strong energy dependence of the differential capture cross section in the forward direction at $k_{\mathrm{f}}=v$ is expected for neutral projectiles.

This contrasts with the results of recent coincidence experiments on the forward peak. Recording the momentum distribution of the ejected electrons simultaneously with the charge state of the outgoing projectile, a procedure that allows for separation between electron loss and capture-to-continuum (CTC) processes, Sarkadi et al (1989) have found that for the collision systems $\mathrm{H}^{\circ}, \mathrm{He}^{\circ}+\mathrm{He}$, Ar a CTC forward peak not only exists, but is even narrower than in the case of charged projectiles. A similar behaviour is also known from electron loss experiments (Duncan and Menendez 1979) where the forward peaks resulting from the impact of negatively charged $\mathrm{H}^{-}$and neutral $\mathrm{H}^{\circ}$ projectiles on argon targets are compared, although these experiments are non-coincident so that double electron loss from $\mathrm{H}^{-}$cannot be excluded.

The variance between theoretical expectation and experiment calls for a careful investigation of the forward peak region for neutral projectiles. So far, theoretical considerations have mostly been confined to general arguments for a finite peak height at $\boldsymbol{k}_{\mathrm{f}}=\boldsymbol{v}$ for fully screened projectiles (McGuire et al 1987). An analytical analysis of the s-wave Jost function for a cut-off Coulomb potential and a Hulthén potential supports this conjecture except for specific values of the potential range parameter where zero-energy resonances cause the continuum-state normalisation constant to 
diverge (Garibotti and Barrachina 1983). Another investigation within a onedimensional model where the short-range atomic potential is replaced by a $\delta$ function (Burgdörfer and Wang 1988) also provides evidence for an enhanced, but finite, electron intensity near $k_{\mathrm{f}}=v$ for high collision velocities.

In the present work, electron emission into the projectile continuum is studied in the framework of the impulse approximation (Briggs 1977). In its prior form, this approximation is applicable to energetic collisions of asymmetric systems, i.e. $Z_{\mathrm{P}} \ll Z_{\mathrm{T}}$ and $v \geq Z_{\mathrm{T}}$ where $Z_{\mathrm{P}}$ and $Z_{\mathrm{T}}$ are the nuclear charge of the projectile and of the target, respectively (for electrons from higher shells $n, Z_{\mathrm{T}}$ should be replaced by $Z_{\mathrm{T}} / n$ ). An additional peaking approximation allows for the extraction of the final-state electronic wavefunction from the transition matrix element and thus for a decoupling of the electronic properties in the projectile field from the process of target ionisation. The analysis of the forward peak region can therefore basically be confined to an investigation of low-energy electron scattering from the projectile. Section 2 reviews the theory for electron scattering from a short-range or (for the sake of comparison) ionic potential, where the presence of bound electrons is accounted for by means of an additional exchange interaction. The potentials and their particular forms for $\mathrm{H}$ and $\mathrm{He}$ are discussed in $\$ 3$. Details of the calculation and numerical results for the projectile scattering states are presented in $\S 4$. The cTC cross section for $1 \mathrm{MeV} / N \mathrm{He}^{0}$ colliding with $\mathrm{Ne}$ is calculated in $\S 5$ and compared with the results for singly $\left(\mathrm{He}^{+}\right)$and doubly $\left(\mathrm{He}^{2+}\right)$ charged projectiles, and the conclusions are drawn in $\S 6$. Atomic units $(\hbar=m=$ $e=1)$ are used unless otherwise indicated.

\section{The scattering problem}

The doubly differential cross section for the ejection of electrons into low-lying continuum states of the projectile may be approximated by

$$
\mathrm{d}^{2} \sigma / \mathrm{d} E_{\mathrm{f}} \mathrm{d} \Omega_{\mathrm{f}}=(2 \pi)^{3}\left|\psi_{\mathrm{f}}^{\mathrm{P}}(\boldsymbol{r}=0)\right|^{2} \sum_{i} \mathrm{~d}^{2} \sigma_{i}^{1} / \mathrm{d} E_{\mathrm{f}} \mathrm{d} \Omega_{\mathrm{f}}
$$

where $\psi_{\mathrm{f}}^{\mathrm{P}}$ is the electronic final projectile state (taken to be $\delta$-normalised in momentum space) and $\mathrm{d}^{2} \sigma_{i}^{\mathrm{l}} / \mathrm{d} E_{\mathrm{f}} \mathrm{d} \Omega_{\mathrm{f}}$ is the doubly differential cross section for the ejection of a target electron from the state $\psi_{i}^{\mathrm{T}}$ into a target continuum state $\psi_{\boldsymbol{k}_{\mathrm{f}}}^{\mathrm{T}}$ with momentum $\boldsymbol{k}_{\mathrm{f}}$ ( $E_{\mathrm{f}}$ and $\mathrm{d} \Omega_{\mathrm{f}}$ being energy and solid angle, respectively, of the emitted electron) given by

$\mathrm{d}^{2} \sigma_{i}^{1} / \mathrm{d} E_{\mathrm{f}} \mathrm{d} \Omega_{\mathrm{f}}=2 \pi N_{i}\left(k_{\mathrm{f}} / v\right) \int \mathrm{d} \boldsymbol{q} \delta\left(E_{\mathrm{f}}-\varepsilon_{i}^{\mathrm{T}}-\boldsymbol{q} v\right)\left|V_{\mathrm{p}}(\boldsymbol{q})\left\langle\psi_{k_{\mathrm{f}}}^{\mathrm{T}}\left|\mathrm{e}^{\mathrm{i} \boldsymbol{q} \boldsymbol{r}}\right| \psi_{i}^{\mathrm{T}}\right\rangle\right|^{2}$

In this expression, $V_{\mathrm{P}}(\boldsymbol{q})$ is the Fourier-transformed projectile field, $\varepsilon_{i}^{\top}$ is the electron energy in the initial state $i$, and the sum $i$ in (2.1) runs over all occupied target subshells with degeneracy $N_{i}$. The factorisation (2.1) can be derived from the fully peaked impulse approximation (Briggs 1977, Jakubassa-Amundsen 1988), but also from a high-energy continuum distorted wave-type formulation (Salin 1969). It should be noted that in this formula, correlations between the target electrons as well as excitation of the spectator target electrons are neglected, and (2.1) also does not account for the additional asymmetry of the forward peak (for charged projectiles), which is related to higher-order perturbative effects. These deficiencies should, however, not change our basic conclusions. 
For fully stripped projectiles, the (outgoing) projectile wavefunction at the origin is simply given by the Coulomb normalisation factor

$(2 \pi)^{3 / 2} \psi_{\mathrm{f}}^{\mathrm{P}}(\boldsymbol{r}=0)=\left[2 \pi \eta /\left(1-\mathrm{e}^{-2 \pi \eta}\right)\right]^{1 / 2} \mathrm{e}^{\mathrm{i} \arg \mathrm{\Gamma}(1+\mathrm{i} \eta)} \quad \eta=Z_{\mathrm{p}} / k$

with $k$ the electron momentum relative to the projectile. For a projectile carrying electrons, the final-state wavefunction has to be calculated from a Schrödinger-type equation. For hydrogen- or helium-like systems this equation reads (Nakanishi and Schrader 1986; $\psi_{k}$ denotes an ingoing projectile scattering state)

$$
\begin{aligned}
{\left[-\frac{1}{2} \Delta+V_{00}(r)\right.} & \left.+V_{\mathrm{pol}}(r)\right] \psi_{k}(\boldsymbol{r})+(-1)^{J}\left\{\left\langle\varphi_{0 i}\left(\boldsymbol{r}^{\prime}\right)\left|e^{2} /\left(\left|\boldsymbol{r}-\boldsymbol{r}^{\prime}\right|\right)\right| \psi_{k}\left(\boldsymbol{r}^{\prime}\right)\right\rangle\right. \\
& \left.-\left(E_{k}-\varepsilon_{0 i}\right)\left\langle\varphi_{0 i}\left(\boldsymbol{r}^{\prime}\right) \mid \psi_{k}\left(\boldsymbol{r}^{\prime}\right)\right\rangle\right\} \varphi_{0 i}(\boldsymbol{r})=E_{k} \psi_{k}(\boldsymbol{r}) .
\end{aligned}
$$

Here $E_{k}=k^{2} / 2, \varphi_{0 i}$ and $\varepsilon_{0 i}$ are the wavefunction and energy, respectively, of an occupied single-particle (Hartree-Fock) state, and $J$ accounts for the fact that the total wavefunction of the $N+1$ electrons (where $N$ is the number of bound electrons) has to be antisymmetric (if $N=1, J=0$ for singlet and $J=1$ for triplet states, while $J=1$ for $N=2$ ). The exchange contribution, the term in curly braces in (2.4), consists of the potential exchange term plus an overlap term that assures orthogonality of the scattering state to the bound state. The potential $V_{00}$ is the static potential, consisting of the nuclear field $-Z_{\mathrm{P}} e^{2} / r$ plus the averaged interaction between the free and the bound electrons (the average being taken over the bound states). $V_{\text {pol }}$ is the dynamic polarisation potential, which accounts for the global effect of atomic (or ionic) excitation and de-excitation during the scattering event. This interpretation is based on the fact that the scattering equation is formally obtained upon expanding the $(N+$ 1)-electron wavefunction in terms of the $N$-electron functions of the projectile. After insertion into the full Schrödinger equation and projection onto the ground-state $N$-electron function, an equation of type (2.4) results if only the elastic channel is retained (Bransden and Coleman 1972, Furness and McCarthy 1973).

Equation (2.4) is most conveniently solved by means of a partial-wave expansion

$$
\begin{aligned}
& \psi_{\boldsymbol{k}}(\boldsymbol{r})=\left[4 \pi /(2 \pi)^{3 / 2}\right] \sum_{l m} \mathrm{i}^{l}\left[R_{l}(k r) / r\right] Y_{l m}^{*}(\hat{\boldsymbol{k}}) Y_{l m}(\hat{\boldsymbol{r}}) \\
& 1 /\left(\left|\boldsymbol{r}-\boldsymbol{r}^{\prime}\right|\right)=4 \pi \sum_{l m}[1 /(2 l+1)]\left(r_{</ r_{>}^{l}}^{l+1}\right) Y_{l m}(\hat{\boldsymbol{r}}) Y_{l m}^{*}\left(\hat{\boldsymbol{r}}^{\prime}\right)
\end{aligned}
$$

where $r_{<}=\min \left(r, r^{\prime}\right), r_{>}=\max \left(r, r^{\prime}\right)$ and $Y_{l m}$ is a spherical harmonic. For the CTC cross section, equation (2.1), knowledge of the wavefunction $\psi_{k}$ at the origin is sufficient. As $R_{l}(k r) \sim r^{l+1}$ for $r \rightarrow 0$, which follows from the Coulombic behaviour at small distances, only the $l=0$ partial wave contributes to $\psi_{k}(r=0)$. For a spherically symmetric state $\varphi_{0 i}(r)$ the equation for $R_{0}(k r)$ reads

$$
\begin{aligned}
{\left[\mathrm{d}^{2} / \mathrm{d} r^{2}-2(\right.} & \left.\left.V_{00}+V_{\mathrm{pol}}\right)+k^{2}\right] R_{0}(k r) \\
= & (-1)^{J} 8 \pi r \varphi_{0 i}(r)\left[(1 / r) \int_{0}^{r} \mathrm{~d} r^{\prime} r^{\prime} \varphi_{0 i}\left(r^{\prime}\right) R_{0}\left(k r^{\prime}\right)\right. \\
& \left.+\int_{r}^{\infty} \mathrm{d} r^{\prime} \varphi_{0 i}\left(r^{\prime}\right) R_{0}\left(k r^{\prime}\right)+\left(\varepsilon_{0 i}-k^{2} / 2\right) \int_{0}^{\infty} \mathrm{d} r^{\prime} r^{\prime} \varphi_{0 i}\left(r^{\prime}\right) R_{0}\left(k r^{\prime}\right)\right]
\end{aligned}
$$

which has to be solved self-consistently. For numerical purposes, it is convenient to transform (2.6) into two coupled first-order equations by means of introducing $\mathrm{d} R_{0} / \mathrm{d} r \equiv p$ as a new variable. 
The asymptotic behaviour of the properly normalised function $R_{0}^{n}$ is determined by

$$
R_{0}^{n}(k r) \rightarrow(1 / k) \mathrm{e}^{\mathrm{i} \delta_{0}} \sin \left\{k r+\delta_{0}+\left[\left(Z_{\mathrm{P}}-N\right) / k\right] \ln (2 k r)\right\} \quad r \rightarrow \infty
$$

where $\delta_{0}$ is the phaseshift and the factor $Z_{\mathrm{P}}-N$ in front of the logarithmic phase is the excess charge of the projectile. If (2.6) is solved with the initial condition $R_{0}(k r)=r$ at $r \rightarrow 0$, the normalisation constant is obtained from

$$
\begin{aligned}
& N_{0} \equiv(2 \pi)^{3 / 2} \psi_{k}(r=0)=\left(1 / R_{\max } k\right) \mathrm{e}^{\mathrm{i} \delta_{0}} \\
& \tan \delta_{0}=\frac{R_{0}\left(k r_{2}\right) \sin \rho_{1}-R_{0}\left(k r_{1}\right) \sin \rho_{2}}{R_{0}\left(k r_{1}\right) \cos \rho_{2}-R_{0}\left(k r_{2}\right) \cos \rho_{1}} \\
& \rho_{1,2}=k r_{1,2}+\frac{Z_{\mathrm{P}}-N}{k} \ln \left(2 k r_{1,2}\right)
\end{aligned}
$$

where $R_{\max }$ is the maximum of $R_{0}(k r)$ in the asymptotic region, and $r_{1}, r_{2}$ are two coordinates in this region.

\section{Choice of the potentials}

The static potential $V_{00}$ for neutral atoms is usually represented by a fit to the Hartree-Fock potential, using for $\mathrm{H}$ and $\mathrm{He}$ the form

$$
V_{00}(r)=-\frac{Z_{\mathrm{P}}}{r}\left(\mathrm{e}^{-\lambda_{1} r}+r \sum_{j=1}^{2} b_{j} \mathrm{e}^{-\mu_{j} r}\right)
$$

where $\lambda_{1}, b_{j}$ and $\mu_{j}$ are fit parameters (Strand and Bonham 1964). For charged projectiles, the residual Coulomb field has to be added, and the simple form may be adopted (Nielsen and Dahler 1977)

$$
V_{00}(r)=-\left(Z_{\mathrm{P}}-N\right) / r-(N / r)\left(1+r / 2 d_{1}\right) \mathrm{e}^{-r / d_{1}}
$$

where $d_{1}$ is chosen to reproduce the correct ground-state binding energy. For $\mathrm{He}^{+}$, one has $d_{1}=0.3045$ (Jakubassa-Amundsen 1983).

As concerns the polarisation potential $V_{\text {pol }}$, a tractable approximation is much more difficult to find, because the only well known property is its long-range behaviour, $V_{\mathrm{pol}} \rightarrow-\alpha_{1} / 2 r^{4}$, where $\alpha_{1}$ is the dipole polarisability $\left(\alpha_{1}=4.5\right.$ for $\mathrm{H}, \alpha_{1}=4.5 / 16$ for $\mathrm{He}^{+}$(Dalgarno and Lynn 1957), and $\alpha_{1}=1.38414$ for $\mathrm{He}^{0}$ (Nesbet 1979)). For highenergy electron scattering, the following form is usually adopted (Jhanwar et al 1978)

$$
V_{\mathrm{pol}}^{(\mathrm{a})}(r)=-\frac{\alpha_{1} r^{2}}{2\left(r^{2}+d^{2}\right)^{3}}-\frac{\alpha_{2} r^{4}}{2\left(r^{2}+d^{2}\right)^{5}} \quad d=\frac{3 k_{0}}{8 \Delta} .
$$

The constant $\alpha_{2}$ is the quadrupole polarisability $\left(\alpha_{2}=15.0\right.$ for $\mathrm{H}$, and $\alpha_{2}=2.32$ for $\mathrm{He}^{0}$ (Jhanwar and Khare 1975)) and $d$ represents the cut-off at small $r$. For scattering energies $E_{k}$ exceeding $3 \Delta$, where $\Delta$ is the mean excitation energy of the atom $(\Delta=0.465$ for $\mathrm{H}$, and $\Delta=1.22$ for $\mathrm{He}^{\mathrm{o}}$ ), the parameter $k_{0}$ can be set equal to the electron momentum $k$ (Jhanwar and Khare 1975). For low-energy scattering, $d$ should be constant. We have used $k_{0}$ as a fit parameter and adjusted it to reproduce the exact phaseshift $\pi / 2$ at the lowest resonance. 
An alternative way to approximate $V_{\text {pol }}$ consists of the introduction of a cut-off function $w(x)$, which is specially adapted to low-energy scattering (Nakanishi and Schrader 1986)

$$
\begin{array}{ll}
V_{\mathrm{pol}}^{(\mathrm{b})}(r)=-\left(\alpha_{1} / 2 r^{4}\right) w(x) & x=\lambda_{n} r / r_{0} \\
w(x)=\left[1-e_{n}(x) \mathrm{e}^{-x}\right]^{2} & e_{n}(x)=\sum_{i=0}^{n} x^{i} / i ! .
\end{array}
$$

The choice of $n=8$ and $\lambda_{n}=10.6172$ follows the suggestion of Nakanishi and Schrader (1986). The effective radius $r_{0}$ is determined from the requirement that the correct scattering length (for neutrals) or binding energy (for ions) is reproduced: it is $r_{0}=1.926$ for $\mathrm{H}\left({ }^{1} \mathrm{~S}\right)$; twice this value, $r_{0}=3.852$ for $\mathrm{H}\left({ }^{3} \mathrm{~S}\right) ; r_{0}=2.373$ for $\mathrm{He}^{0}$; and $r_{0}=1.795$ for $\mathrm{He}^{+}\left({ }^{3} \mathrm{~S}\right)$. For energies $E_{k}$ below the first ionisation threshold, the potential (3.4) leads to phaseshifts that are in general closer to the ones resulting from $a b$ initio calculations than the phaseshifts obtained with $V_{\text {pol }}^{(\mathrm{a})}$.

\section{Numerical solution of the scattering equation}

We have solved the two coupled equations for $R_{0}(r)$ and $p(r)$ which derive from (2.6) by means of the Runge-Kutta method, taking logarithmic steps for small $r$ and linear steps for larger values of $r$. Self-consistency is achieved in the following way. In a first step, the exchange term (i.e. the RHS of (2.6)) is put equal to zero, and the two integrals

$$
\int_{0}^{\infty} d r^{\prime} r^{\prime n} \varphi_{0 i}\left(r^{\prime}\right) R_{0}\left(k r^{\prime}\right)
$$

with $n=0,1$ are calculated in a zeroth approximation (the integral from $r$ to $\infty$ on the RHS of equation (2.6) is split into one from 0 to $\infty$ and one from 0 to $r$ ). The wavefunction $\varphi_{0 i}$ is taken to be a Clementi-Roetti function for $\mathrm{He}^{0}$ (Clementi and Roetti 1974, Jakubassa-Amundsen 1983) while it is hydrogenic for $\mathrm{H}$ and $\mathrm{He}^{+}$. In the second step, the exchange term is fully included by doing the finite integrals

$$
\int_{0}^{r} \mathrm{~d} r^{\prime} r^{\prime n} \varphi_{0 i}\left(r^{\prime}\right) R_{0}\left(k r^{\prime}\right)
$$

simultaneously with the differential equations, and by using the zeroth approximation for the two infinite integrals. With the improved $R_{0}$, the infinite integrals are calculated anew and the iteration is continued until convergence is obtained.

For numerical reasons we have also investigated the importance of the overlap term (the last term in (2.6)). We have found that for hydrogen singlet states the inclusion of this term is crucial for reproducing the strong $k$ dependence of the phaseshift. In fact, orthogonalisation is particularly important for electrons in a spatially symmetric state that allows for small interelectronic distances, and only properly orthogonalised states do carry the signature of the bound singlet state in $\mathrm{H}^{-}$ that is responsible for the rapid variation of the phaseshift with $k$ (Wu and Ohmura 1962). On the other hand, for helium or hydrogen triplet states, the overlap term is very small when calculated non-self-consistently (i.e. when calculated with an $R_{0}$ where only the potential exchange term is retained), which means that, to a good approximation, the scattering function is from the outset orthogonal to the bound state. We have 
Table 1. Phaseshift $\delta_{0}$ (modulo $\pi$ ) and modulus $\left|N_{0}\right|$ of the normalisation constant for the $l=0$ partial wave of an electron with momentum $k$ scattering from neutral hydrogen (columns 2-7): s denotes spin singlet; $t$ denotes triplet state; (a) denotes use of potential $V_{\text {pol }}^{(\text {a) }}$ (equation (3.3)); (b) results from $V_{\text {pol }}^{(\text {b) }}$ (equation (3.4)). The last column shows $\left|N_{0}\right|$ for a Coulomb wave to charge $Z_{\mathrm{P}}=1$ (equation (2.3)). Atomic units are used.

\begin{tabular}{|c|c|c|c|c|c|c|c|}
\hline \multirow[b]{2}{*}{$k$} & \multicolumn{2}{|c|}{$\mathrm{H}^{0}\left({ }^{1} \mathrm{~S}\right)$} & \multicolumn{4}{|c|}{$\mathrm{H}^{0}\left({ }^{3} \mathrm{~S}\right)$} & \multirow{2}{*}{$\frac{\mathrm{H}^{+}}{\left|N_{0}\right|^{\text {cou } !}}$} \\
\hline & $\delta_{0}^{\mathrm{s}(\mathrm{b})}$ & $\left|N_{0}\right|^{s(b)}$ & $\delta_{0}^{\mathrm{t}(\mathrm{a})}$ & $\left|N_{0}\right|^{(a)}$ & $\delta_{0}^{1(b)}$ & $\left|N_{0}\right|^{t(b)}$ & \\
\hline 0.001 & -0.0060 & 2.944 & -0.0014 & 3.323 & -0.0017 & 2.852 & 79.267 \\
\hline 0.01 & -0.0601 & 2.939 & -0.0144 & 3.320 & -0.0177 & 2.851 & 25.066 \\
\hline 0.05 & -0.3013 & 2.882 & -0.0786 & 3.285 & -0.0948 & 2.817 & 11.210 \\
\hline 0.1 & -0.5891 & 2.737 & -0.1690 & 3.223 & -0.1994 & 2.774 & 7.927 \\
\hline 0.2 & -1.0789 & 2.373 & -0.3644 & 3.087 & -0.4185 & 2.690 & 5.605 \\
\hline 0.3 & -1.4535 & 2.071 & -0.5653 & 2.949 & -0.6352 & 2.608 & 4.577 \\
\hline 0.4 & 1.4002 & 1.875 & -0.7640 & 2.811 & -0.8416 & 2.532 & 3.963 \\
\hline 0.5 & 1.1754 & 1.766 & -0.9559 & 2.677 & -1.0321 & 2.455 & 3.545 \\
\hline 0.6 & 0.9991 & 1.718 & -1.1389 & 2.549 & -1.2053 & 2.383 & 3.236 \\
\hline 0.7 & 0.8642 & 1.711 & -1.3114 & 2.428 & -1.3609 & 2.312 & 2.996 \\
\hline 0.75 & 0.8096 & 1.719 & -1.3944 & 2.370 & -1.4321 & 2.278 & 2.895 \\
\hline 0.8 & 0.7630 & 1.732 & -1.4743 & 2.316 & -1.4991 & 2.245 & 2.803 \\
\hline 0.8369 & 0.7323 & 1.744 & -1.5324 & 2.276 & -1.5462 & 2.221 & 2.741 \\
\hline 0.862 & & & -1.57 & 2.250 & 1.5649 & 2.205 & 2.701 \\
\hline
\end{tabular}

Table 2. Phaseshift $\delta_{0}$ and modulus $\left|N_{0}\right|$ of the normalisation constant for the $l=0$ partial wave of an electron with momentum $k$ scattering from neutral $\mathrm{He}$ (columns 2-5) and triplet $\mathrm{He}^{+}$(columns 6-7): (a) denotes use of the potential $V_{\text {pol }}^{(\mathrm{a})}$ (equation (3.3)); (b) results from $V_{\text {pol }}^{(\mathrm{b})}$ (equation (3.4)).

\begin{tabular}{|c|c|c|c|c|c|c|}
\hline \multirow[b]{2}{*}{$k$} & \multicolumn{4}{|c|}{$\mathrm{He}^{0}$} & \multicolumn{2}{|c|}{$\mathrm{He}^{+}\left({ }^{3} \mathrm{~S}\right)$} \\
\hline & $\delta_{0}^{(\mathrm{a})}$ & $\left|N_{0}\right|^{(a)}$ & $\delta_{0}^{(b)}$ & $\left|N_{\mathrm{o}}\right|^{(\mathrm{b})}$ & $\delta_{0}^{(\mathrm{b})}$ & $\left|\boldsymbol{N}_{0}\right|^{(b)}$ \\
\hline 0.001 & -0.0013 & 3.564 & -0.0012 & 3.724 & & \\
\hline 0.01 & -0.0128 & 3.563 & -0.0119 & 3.723 & & \\
\hline 0.05 & -0.0660 & 3.549 & -0.0617 & 3.708 & & \\
\hline 0.1 & -0.1360 & 3.523 & -0.1274 & 3.681 & & \\
\hline 0.2 & -0.2815 & 3.456 & -0.2641 & 3.616 & 0.3330 & 6.782 \\
\hline 0.3 & -0.4294 & 3.381 & -0.4025 & 3.544 & -0.4481 & 5.541 \\
\hline 0.4 & -0.5762 & 3.299 & -0.5387 & 3.470 & 0.4459 & 4.817 \\
\hline 0.5 & -0.7196 & 3.212 & -0.6700 & 3.391 & 0.8496 & 4.329 \\
\hline 0.6 & -0.8583 & 3.123 & -0.7952 & 3.309 & 1.0760 & 3.972 \\
\hline 0.7 & -0.9917 & 3.031 & -0.9133 & 3.227 & 1.1746 & 3.701 \\
\hline 0.8 & -1.1192 & 2.939 & -1.0239 & 3.145 & 1.2423 & 3.484 \\
\hline 0.9 & -1.2409 & 2.849 & -1.1268 & 3.063 & 1.2862 & 3.306 \\
\hline 1.0 & -1.3567 & 2.760 & -1.2222 & 2.983 & 1.3197 & 3.155 \\
\hline 1.1 & -1.4668 & 2.674 & -1.3102 & 2.906 & 1.3498 & 3.026 \\
\hline 1.194 & -1.57 & 2.596 & -1.3873 & 2.835 & 1.3803 & 2.920 \\
\hline
\end{tabular}


therefore omitted the overlap term in the case of $J=1$, which speeds up convergence considerably.

The modulus $\left|N_{0}\right|$ of the normalisation constant as well as the phaseshifts for electron scattering on hydrogen and helium, respectively, are listed in tables 1 and 2 as a function of the electron momentum $k$. In order to check the reliability of the calculated values of $N_{0}$, we have performed the runs for $\mathrm{H}\left({ }^{3} \mathrm{~S}\right)$ and $\mathrm{He}^{0}$ with both polarisation potentials $V_{\mathrm{pol}}^{(\mathrm{a})}$ and $V_{\mathrm{pol}}^{(\mathrm{b})}$. The parameter $k_{0}$ in $V_{\mathrm{pol}}^{(\mathrm{a})}$ is taken to be $k_{0}=0.6684$ for $\mathrm{H}\left({ }^{3} \mathrm{~S}\right)$ and $k_{0}=1.107$ for $\mathrm{He}^{0}$ in order to reproduce the phaseshift $\delta_{0}=\pi / 2$ at the resonance position $k=0.862$ and $k=1.194$ for $\mathrm{H}\left({ }^{3} \mathrm{~S}\right)$ and $\mathrm{He}^{0}$, respectively. The difference in $\left|N_{0}\right|$ for the two potentials $V_{\text {pol }}^{(a)}$ and $V_{\text {pol }}^{(b)}$ is in most cases smaller than $10 \%$. Since the phaseshifts from $V_{\text {pol }}^{(\text {b) }}$ are closer to the exact phaseshifts from $a b$ initio calculations (Rudge 1975, Nesbet 1979), we have only used this potential for the other cases, $\mathrm{H}\left({ }^{1} \mathrm{~S}\right)$ and $\mathrm{He}^{+}\left({ }^{3} \mathrm{~S}\right)$. It should be noted, however, that even a model potential of the type (3.4) is unable to describe the hydrogen singlet resonance at $k=0.8369$ since electron-electron correlations are not taken into account (Michels and Harris 1967). We have also studied the behaviour of $N_{0}$ if the parameters $k_{0}$ and $r_{0}$ are fitted at each momentum $k$ so as to reproduce the exact phaseshifts. In most cases, the deviations of $\left|N_{0}\right|$ from the tabulated values are well below $2 \%$, the only exception being for $\mathrm{H}\left({ }^{3} \mathrm{~S}\right)$ when calculated with $V_{\text {pol }}^{(a)}(\leqslant 5 \%)$. We thus conclude that the values $\left|N_{0}\right|^{(b)}$ represent the 'best' values with an accuracy of about $10 \%$ (the numerical accuracy is well below $1 \%$ ).

For the charged projectile, $\mathrm{He}^{+}$, the phaseshift $\delta_{0}$ is dominated for $k \rightarrow 0$ by the strongly varying Coulomb phaseshift, and so is the normalisation constant. We have found, however, that the ratio between $\left|N_{0}\right|$ and the Coulomb normalisation $\left|N_{0}\right|^{\text {coul }} \simeq$ $(2 \pi / k)^{1 / 2}$, given in the last column of table 1 , approaches a constant, $\left|N_{0}\right| /\left|N_{0}\right|^{\text {coul }} \approx$ 1.21 , for $k \leqslant 0.2$. One may thus use this relation for calculating $\left|N_{0}\right|$ at $k \leqslant 0.1$ rather than the scattering equation where the asymptotic region is not reached until very large $r(r>4000$ au for $k \leqslant 0.1)$.

\section{Calculation of the capture cross section}

For comparison with experimental electron spectra, the doubly differential cross section (2.1) has to be averaged over the detector resolution. Since the energy resolution is in general rather good, the cross section for electron emission in the beam direction $\left(\vartheta_{\mathrm{f}}=0^{\circ}\right.$ ) is predominantly affected by the average over the angular resolution $\theta_{0}$

$$
\left\langle\frac{\mathrm{d}^{2} \sigma}{\mathrm{d} E_{\mathrm{f}} \mathrm{d} \Omega_{\mathrm{f}}}\right\rangle_{\theta_{\mathrm{o}}}=\frac{1}{1-\cos \theta_{0}} \int_{0}^{\theta_{0}}\left(\sin \vartheta_{\mathrm{f}}\right) \mathrm{d} \vartheta_{\mathrm{f}} \frac{\mathrm{d}^{2} \sigma}{\mathrm{d} E_{\mathrm{f}} \mathrm{d} \Omega_{\mathrm{f}}}
$$

The ionisation cross section $\mathrm{d}^{2} \sigma_{i}^{1} / \mathrm{d} E_{\mathrm{f}} \mathrm{d} \Omega_{\mathrm{f}}$ (equation (2.2)) is a smooth function of $\vartheta_{\mathrm{f}}$ in the region of interest, and the integration (5.1) will mainly operate on the prefactor in (2.1), the squared normalisation constant of the final projectile state. In order to demonstrate the effect of the angular average on the electron spectrum, we have shown in figure 1 the average of the squared normalisation constant alone, $\left|N_{0}\right|_{\theta_{0}}^{2}$, for a neutral and a charged hydrogen projectile. In the case of $\mathrm{H}^{+}$, where without averaging $N_{0}$ would be infinite for the electron momentum $k_{\mathrm{f}}=v$, a narrow peak is seen, its width increasing proportional to $\theta_{0}$ (Dettmann et al 1974). For $\mathrm{H}^{0}$, on the other hand, the increase of intensity towards $k_{\mathrm{f}}=v$ is due to the long-range attractive polarisation potential and is thus much weaker than for the Coulomb field. However, exchange 


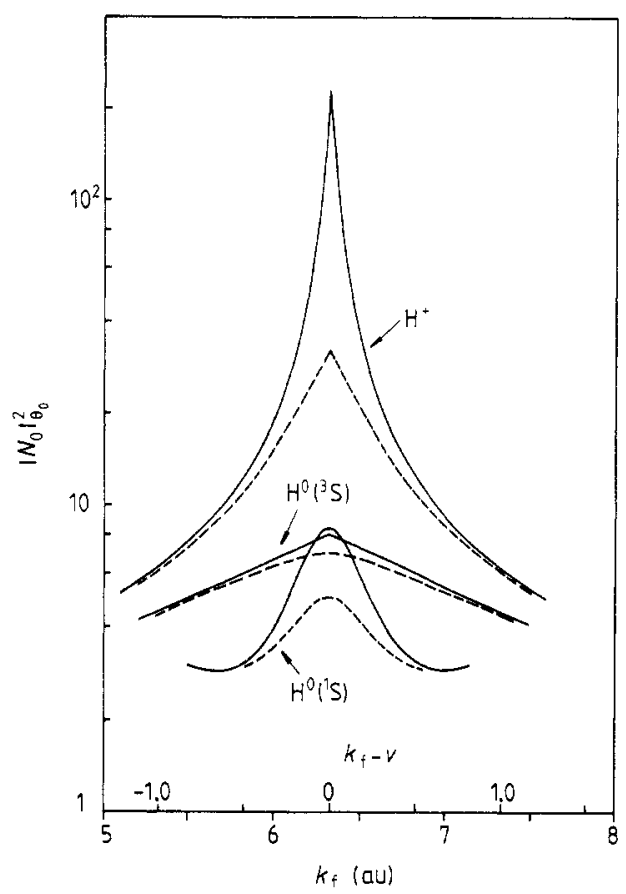

Figure 1. Angular-averaged squared normalisation constant of the projectile state as a function of electron momentum $k_{f}$ for protons and hydrogen in the spin singlet ( ${ }^{1} S$ ) and triplet $\left({ }^{3} \mathrm{~S}\right)$ states, respectively. The collision velocity is $v=6.328 \mathrm{au}$, and the resolution is $\theta_{0}=0.5^{\circ}\left(v \theta_{0}=3.164\right.$, full curves $)$ and $\theta_{0}=3.5^{\circ}\left(v \theta_{0}=22.15\right.$; broken curves $)$.

symmetry also influences the energy distribution of the electrons by means of the additional non-local (though short-range) potential. While, for the triplet state, the peak is very broad and hence hardly dependent on the resolution $\theta_{0}$, a rather pronounced peak is present for the singlet state, although its width still exceeds that of the Coulomb peak. The second increase of the singlet intensity for $\left|k_{\mathrm{f}}-v\right| \geqslant 0.8$ marks the onset of the resonance at $k=0.8369$.

The strong enhancement of the singlet intensity for $k_{\mathrm{f}} \rightarrow v$, which is related to the rapid decrease of the phaseshift with momentum $k$ for $k \ll 1$, results from the presence of a singlet bound state in $\mathrm{H}^{-}$lying close to threshold. On the other hand, no bound triplet $\mathrm{H}^{-}$state exists, and hence the phaseshift for the triplet state varies slowly with $k$ as does the normalisation constant $N_{0}$.

Although the curves in figure 1 are obtained for a collision velocity $v=6.328$ (corresponding to $1 \mathrm{MeV} / N$ ) at the two angular resolutions $\theta_{0}=0.5^{\circ}$ and $3.5^{\circ}$, they actually depend to a very good approximation only on the single parameter $v \theta_{0}$ if plotted versus $k_{\mathrm{f}}-v$, due to the scaling properties of the averaging procedure. The scaling is easily seen by means of changing the integration variable in (5.1) from $\vartheta_{f}$ to $k=\left(k_{\mathrm{f}}^{2}+v^{2}-2 k_{\mathrm{f}} v \cos \vartheta_{\mathrm{f}}\right)^{1 / 2}$ and by recalling that $k_{\mathrm{f}}=v$ and $\cos \vartheta_{\mathrm{f}} \simeq 1-\vartheta_{\mathrm{f}}^{2} / 2$.

For the calculation of the ionisation cross section (2.2), which is required for the proper evaluation of (5.1), we have used hydrogenic target wavefunctions with Slaterscreened effective charge and experimental binding energies, allowing us to perform the transition matrix elements analytically (Jakubassa-Amundsen 1988). For the projectile field entering into (2.2) we have used the high-energy approximation to the potentials 
given in (2.4) by neglecting the exchange contribution and by representing the polarisation potential by the version (3.3) with $k_{0}=v$. For $\mathrm{He}^{0}$, the Fourier transform of the projectile field $V_{00}+V_{\text {pol }}$ reads

$$
\begin{aligned}
& V_{\mathrm{P}}(\boldsymbol{q})=-Z_{\mathrm{P}}\left(\frac{2}{\pi}\right)^{1 / 2}\left(\frac{1}{q^{2}+\lambda_{1}^{2}}+\sum_{j=1}^{2} \frac{2 \mu_{j} b_{j}}{\left(q^{2}+\mu_{j}^{2}\right)^{2}}\right)+V_{\alpha_{1}}(\boldsymbol{q})+V_{\alpha_{2}}(\boldsymbol{q}) \\
& V_{\alpha_{1}}(\boldsymbol{q})=-\frac{\alpha_{1}}{16 d}\left(\frac{\pi}{2}\right)^{1 / 2}(3-q d) \mathrm{e}^{-q d} \\
& V_{\alpha_{2}}(\boldsymbol{q})=-\frac{\alpha_{2}}{768}\left(\frac{\pi}{2}\right)^{1 / 2} \mathrm{e}^{-q d}\left(q^{3}-\frac{10 q^{2}}{d}+\frac{15 q}{d^{2}}+\frac{15}{d^{3}}\right) .
\end{aligned}
$$

The high-energy approximation is justified because, in the projectile reference frame, the target electron in its initial state moves on average with the collision velocity $v$ (which is large compared to the mean excitation energy of the projectile). Moreover, since CTC requires a large momentum transfer $q$, the contribution from the polarisation field is very small (we have neglected this contribution in the case of $\mathrm{He}^{+}$) and $V_{\mathrm{P}}(\boldsymbol{q})$ acts very much like a pure Coulomb field of charge $Z_{\mathrm{p}}$.

Figure 2 shows the angular-averaged differential cross section for capture to the continuum in $1 \mathrm{MeV} / N \mathrm{He}+\mathrm{Ne}$ collisions. The angular resolution is $\theta_{0}=1^{\circ}$ and the contribution of all target electrons is taken into account (the $2 \mathrm{~s}$ and the $2 \mathrm{p}, m=0$ electrons being the most important ones at $v=6.328$ ). The asymmetry of the forward peak is exclusively due to the underlying ionisation cross section, while the prefactor $\left|N_{0}\right|^{2}$ is symmetric (cf figure 1), as a result of the full peaking approximation inherent

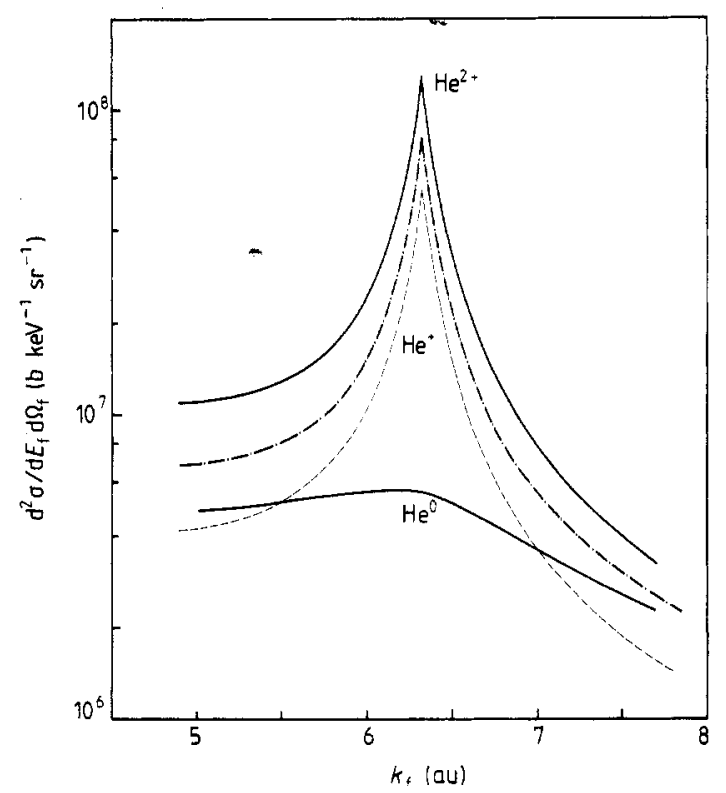

Figure 2. Angular-averaged doubly differential cross section for electron capture to continuum in $1 \mathrm{MeV} / N \mathrm{He}+\mathrm{Ne}$ collisions as a function of electron momentum $k_{\mathrm{f}}$. The resolution is $\theta_{0}=1^{\circ}$. Shown are the results for neutral and fully stripped He (full curves) as well as for singly charged $\mathrm{He}$ in ti.e ${ }^{3} \mathrm{~S}$ state if the ionic projectile field is fully included in the normalisation $N_{0}$ (chain curve) and if $N_{0}$ is calculated from a pure Coulomb field with $Z=1$ (broken curve). 
in the formula (2.1). All three charge states of the projectile are considered: $\mathrm{He}^{0}$, $\mathrm{He}^{+}\left({ }^{3} \mathrm{~S}\right)$ and $\mathrm{He}^{2+}$. It is seen that the intensity of the CTC electrons increases monotonically with increasing projectile field strength at all values of momentum $k_{\mathrm{f}}$ in the peak region, as expected from general considerations. In order to obtain this behaviour it is, however, crucial to calculate the normalisation $N_{0}$ for an ionic projectile from the proper scattering equation. The simple insertion of the Coulomb factor (2.3) for the ionic charge underestimates the cross section considerably and even falls below the $\mathrm{He}^{0}$ result in the tails of the peak (cf figure 2).

\section{Conclusions}

We have calculated the spectrum of target electrons captured into the continuum of neutral ground-state projectiles and have compared with the results for charged projectiles. We have established the existence of a forward peak for neutral projectiles and have found that its shape depends strongly on the spatial symmetry of the total electronic wavefunction. For hydrogen projectiles, the singlet electronic state has a much narrower peak than the (spatial antisymmetric) triplet state. The dependence of the peak width on the detector angular resolution $\theta_{0}$ is, however, much weaker than in case of charged projectiles, and, in contrast to the latter, the width becomes independent of $\theta_{0}$ in the limit $\theta_{0} \rightarrow 0$. This behaviour is ascribed to the short range of the projectile field.

In case of charged, but not fully stripped, projectiles, the spectrum of the CTC electrons is much like the one that would be obtained if the projectile field were replaced by a pure Coulomb field relating to the ionic charge. As anticipated from the existence of a peak for neutrals, the correct incorporation of the short-range ionic potential leads, however, to an additional increase of the CTC electron intensity (about $30 \%$ in the case of $\mathrm{He}^{+}$).

As concerns the experiment on $300 \mathrm{keV} \mathrm{He}^{\circ}+\mathrm{He}, \mathrm{Ar}$ collisions (Sarkadi et al 1989), our results do not support the presence of a forward peak with a width that is comparable to the width for a $\mathrm{He}^{+}$projectile. Although the collision velocity of $v=1.73$ is far too low to rely on a quantitative estimate based on the impulse approximation, the qualitative prediction of a broad peak for neutral ground-state atoms should hold independently of $v$.

There remains the idea from Barrachina (1989) that a projectile that is excited prior to electron capture may support a near-zero energy resonance, causing a sharp forward peak. However, we estimate the excitation probability to be too small to account for the large CTC fractions found in experiments with $\mathrm{H}^{0}$ and $\mathrm{He}^{0}$ projectiles.

\section{Acknowledgments}

I should like to thank P A Amundsen for helpful discussions and the GSI Darmstadt for financial support.

\section{References}

Barrachina R O 1989 Phys. Rev. Lett. submitted Bransden B H and Coleman J P 1972 J. Phys. B: At. Mol. Phys. 5537 
Briggs J S 1977 J. Phys. B: At. Mol. Phys. 103075

Burgdörfer J and Wang J 1988 Phys. Rev. A 384919

Clementi E and Roetti C 1974 At. Data Nucl. Data Tables 14177

Dalgarno A and Lynn N 1957 Proc. Phys. Soc. 70A 2203

Dettmann K, Harrison K G and Lucas M W 1974 J. Phys. B: At. Mol. Phys. 7269

Duncan M M and Menendez M G 1979 Phys. Rev. A 1949

Furness J B and McCarthy I E 1973 J. Phys. B: At. Mol. Phys. 62280

Garibotti C R and Barrachina R O 1983 Phys. Rev. A 282792

Jakubassa-Amundsen D H 1983 J. Phys. B: At. Mol. Phys. 161767

- 1988 Phys. Rev. A 3870

Jhanwar B L and Khare S P 1975 J. Phys. B: At. Mol. Phys. 82659

Jhanwar B L, Khare S P and Kumar A Jr 1978 J. Phys. B: At. Mol. Phys. 11887

Macek J 1970 Phys. Rev. A 1235

McGuire J H, Reeves T, Deb N C and Sil N C 1987 Nucl. Instrum. Methods B 24/25 243

Michels H H and Harris F E 1967 Phys. Rev. Lett. 19885

Nakanishi H and Schrader D M 1986 Phys. Rev. A 341810

Nesbet R K 1979 Phys. Rev. A 2058

Nielsen S E and Dahler J S 1977 Phys. Rev. A 16563

Rudge M R H 1975 J. Phys. B: At. Mol. Phys. 8940

Salin A 1969 J. Phys. B: At. Mol. Phys. 2631

Sarkadi L, Pálinkás J, Kövér A, Berényi D and Vajnai T 1989 Phys. Rev. Lett. 62527

Strand T G and Bonham R A 1964 J. Chem. Phys. 401686

Wu T Y and Ohmura T 1962 Quantum Theory of Scattering (London: Prentice-Hall) p 69 